\title{
Thermalization in the initial stage of heavy ion collisions
}

\author{
Yan Zhu ${ }^{1,2, a}$ \\ ${ }^{1}$ Department of Physics, P.O. Box 35, 40014 University of Jyväskylä, Finland \\ ${ }^{2}$ Helsinki Institute of Physics, P.O. Box 64, 00014 University of Helsinki, Finland
}

\begin{abstract}
The high density non-abelian matter produced in heavy ion collisions is extremely anisotropic. Prethermal dynamics for the anisotropic and weakly coupled matter is discussed. Thermalization is realized with the effective kinetic theory in the leading order accuracy of the weakly coupled expansion. With the initial condition from color glass condensate, hydrodynamization time for the LHC energies is realized to be about 1 $\mathrm{fm} / \mathrm{c}$, while the thermalization happens much later than the hydrodynamization.
\end{abstract}

\section{Introduction}

Many efforts have been made to study the strongly coupled matter created in heavy ion collisions at RHIC and the LHC. Experimentally, measurements of products from high energy heavy ion collisions provide signals from the strongly interaction matter. The theoretical understanding of the strongly interaction matter is, however, involved in the full understanding of the whole process in the experiment, which starts from the accelerating of nucleus, followed by the hadron-parton and parton-hadron phase transitions, and ends up with final state hadrons.

From successful explanation of experimental data, we have revealed many mistreries in the process of QGP creation and effects it resulting in, but many of them still remain to be revealed. Prethermal dynamics towards formation of QGP is one of the less understand puzzles which was devoted to by many physicists for many years. It is well-known that hydrodynamics with small viscosity has been proven to be the correct theory for the spacetime evolution of quark-gluon plasma (QGP), where the strongly interacting matter is locally thermalized. However, it is unable to describe the matter at the very beginning of heavy ion collisions as the anisotropy of the deconfined matter is extremely large. The question of how QGP is formed has puzzled physicists for many years. Classical Yang-Mills (CYM) theory stands to be valid at this stage, but couldn't drive the anisotropic matter to thermalization. Prethermal evolution of the overoccupied and anisotropic status to thermalization was study by the collision of two planer shock waves in supersymmetric $N=4$ Yang-Mills theory [1]. A QCD based prethermal evolution is, however, less constructed, even though the parametric estimation of 'bottom-up' thermalization [2] was realized more than two decades ago.

In the course of the sequential evolution, the formation of QGP has undergone rich processes which include changing in the degree of freedom. At the very beginning of the heavy ion collisions, where soft gluon density is enhanced, classical Yang Mills theory is applicable to describe such a system. Unlike in usual perturbation theory, the expansion of strongly color fields $A_{\mu} \sim 1 / g$ instead

ae-mail: yan.zhu@jyu.fi 
of the coupling parameter $g$ admits a leading order effective theory to 't Hooft coupling $\lambda=g^{2} N_{c}$. It's vital to color glass condensate (CGC) effective theory derived in the framework of classical YangMills theory. Indeed, CGC effective theory provides the initial distribution of soft gluons in high energy heavy ion collisions, in which saturation scale $Q_{s}$ is the typical transverse momentum of soft gluons, and the gluon density is at the oder of $f \sim 1 / \lambda$. The evolution of the overoccupied status is figured out by solving the classical Yang-Mills equations. It is found that the classical fields at the collision point $\tau=0$ is strongly coherent, that the longitudinal components of chromoelectric and chromomagnetic fields are very large while the transverse components vanish [3]. It leads to the energy momentum tensor with negative longitudinal component, i.e. $T_{\mu v}=\operatorname{diag}(\epsilon, p, p,-p)$ versus $T_{\mu \nu}=\operatorname{diag}(\epsilon, p, p, p)$ for a perfect fluid. The system with negative longitudinal pressure is highly unstable, and the coherence of fields is get rid of by quantum fluctuations of color fields in a short time of $\tau \sim 1 / Q_{s}$. The continue evolution of classical fields admits a self-similar behaviour in the distribution function of soft gluons [4]. It, in other words, indicates that the overoccupied system produced in high energy heavy ion collisions can never approach thermalization in the framework of classical Yang-Mills theory.

On the other hand, from the successful description of collective behaviours of soft particles, hydrodynamic description of locally thermalized plasma, i.e., quark-gluon plasma (QGP), has been proven to be the correct theory. However, the initial condition of hydrodynamics, for example the initial equation, and initial time to start hydrodynamical evolution which is also referred to as hydrodynamization time, for the evolution of QGP is still ambiguous, since neither the validity of hydrodynamics can be extend to describe the very anisotropic glasma at the beginning of heavy ion collisions, nor classical Yang-Mills thoery can drive the anisotropic and overoccupied system to thermalization.

Fortunately, the effective kinetic theory (EKT) [5] derived from weak coupling expansion of QCD can extend the evolution of the system from the overoccupied to underoccupied status as long as the occupancy is not nonperturbatively large $f \ll 1 / \lambda$ and the momentum of gluons is significantly larger than the in-medium screening scale $p^{2}>m^{2} \equiv \lambda \int_{\mathbf{p}} f(p) / p$. Therefore, the EKT and CYM theory provide equivalent description in a wide range of $1 \ll f \ll 1 / \lambda$ for a weakly coupled QCD matter.

In this proceeding, we will discuss prethermal processes through QCD in a weakly coupled plasma. The EKT will be introduced in section 2 before using it to bridge the gap between classical Yang-Mills theory and hydrodynamics. Prethermal evolution from weakly coupled glasma will be discussed in section 3. We will close the discussion in section 4.

\section{The effective kinetic theory}

The leading order EKT [5] is expressed as the effective Boltzmann equation for the color and spin averaged gluon distribution function. With the inclusion of longitudinal expansion, it is written as

$$
-\left(\frac{d f_{\mathbf{p}}}{d \tau}-\frac{p_{z}}{\tau} \frac{\partial}{\partial p_{z}} f(\mathbf{p})\right)=C_{1 \leftrightarrow 2}\left[f_{\mathbf{p}}\right]+C_{2 \leftrightarrow 2}\left[f_{\mathbf{p}}\right] .
$$

We have assumed that the space distribution of gluons is isotropic and uniform, and the momentum distribution is symmetric on the transverse plane but can be anisotropic for the longitudinal momentum along z-direction so that it can be specified as $f_{\mathbf{p}}=f_{x_{p}, p}$ with $x_{p} \equiv \hat{\mathbf{z}} \cdot \hat{\mathbf{p}}$. The collision terms are give by

$$
\begin{aligned}
C_{2 \leftrightarrow 2}[f](\tilde{\mathbf{p}}) & =\frac{(2 \pi)^{3}}{4 \pi \tilde{p}^{2}} \frac{1}{8 v} \int d \Gamma_{P S}|\mathcal{M}|^{2}\left(f_{\mathbf{p}} f_{\mathbf{k}} g_{\mathbf{p}^{\prime}} g_{\mathbf{k}^{\prime}}-f_{\mathbf{p}^{\prime}} f_{\mathbf{k}^{\prime}} g_{\mathbf{p}} g_{\mathbf{k}}\right) \\
& \times\left[\delta(\tilde{\mathbf{p}}-\mathbf{p})+\delta(\tilde{\mathbf{p}}-\mathbf{k})-\delta\left(\tilde{\mathbf{p}}-\mathbf{p}^{\prime}\right)-\delta\left(\tilde{\mathbf{p}}-\mathbf{k}^{\prime}\right)\right]
\end{aligned}
$$



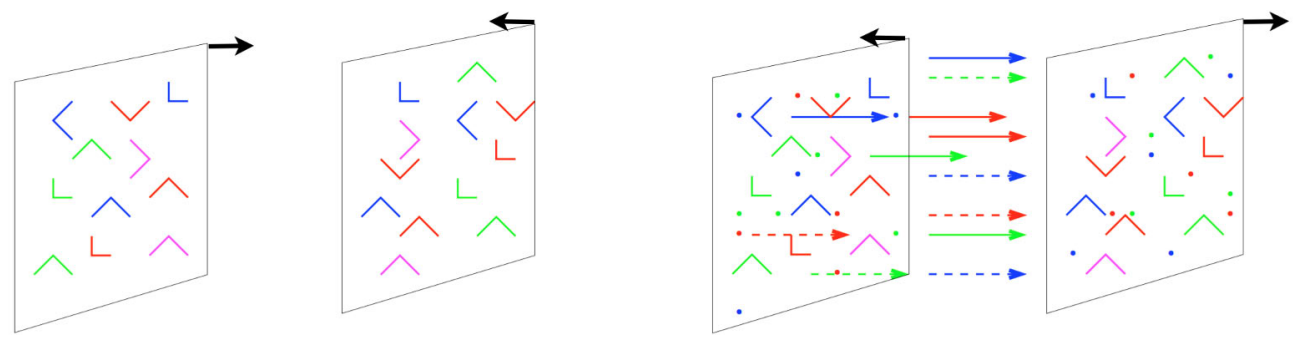

Figure 1. Color fields before (left) and after (right) collisions (figures taken from [3]). Transverse fields on the Lorentz contracted nucleus before collisions, and longitudinal fields are produced after the collision.

where $v=2 d_{A}, g_{\mathbf{p}} \equiv 1+f_{\mathbf{p}}$ and $\frac{|\mathcal{M}|^{2}}{16 \lambda^{2} d_{A}}=\left(\frac{9}{4}+\frac{(s-t)^{2}}{u^{2}}+\frac{(u-s)^{2}}{t^{2}}+\frac{(t-u)^{2}}{s^{2}}\right) . d \Gamma_{P S}$ is the integral measure over the phase space of $2 \leftrightarrow 2$ processes, and the collinear splitting process is expressed

$$
\begin{aligned}
C_{1 \leftrightarrow 2}[f](\tilde{\mathbf{p}}) & =\frac{(2 \pi)^{3}}{4 \pi \tilde{p}^{2}} \frac{1}{v} \int_{0}^{\infty} d p \int_{0}^{p / 2} d k^{\prime}\left[4 \pi \gamma\left(p ; p^{\prime}, k^{\prime}\right)\right]\left(f_{x_{p}, p} g_{x_{p}, p^{\prime}} g_{x_{p}, k^{\prime}}-g_{x_{p}, p} f_{x_{p}, p^{\prime}} f_{x_{p}, k^{\prime}}\right) \\
& \times\left[\delta(\tilde{p}-p)-\delta\left(\tilde{p}-p^{\prime}\right)-\delta\left(\tilde{p}-k^{\prime}\right)\right],
\end{aligned}
$$

where $p^{\prime}=p-k^{\prime}$, and $\gamma\left(p ; p^{\prime}, k^{\prime}\right)$ is the effective collinear splitting rate. The infrared divergence in elastic and inelastic scatterings are regulated by the hard thermal loop resummed screening such that $1 / q^{2} \rightarrow 1 /\left(q^{2}+m^{2}\right)$. Currently, we used isotropic screening in the calculation. Thus Weibel instabilities [6] in the beginning of prethermal evolution are neglected. From the numerical simulation of classical Yang-Mills theory, it is found that the effect of instabilities is only visible in the beginning of the prethermal evolution [7]. Numerical results of the EKT on a 2+1D grid will be discussed later.

\section{Initial stages and bottom-up thermalization}

The CYM description of heavy ion collision is depicted in figure 1. The fields are distributed on the transverse plan of the Lorentz contracted nucleus before collisions. Solving the Yang-Mills equation near the light cone shows that the transverse components of colorelectric and colormagnetic fields vanish at $\tau \rightarrow 0$, whereas the longitudinal components are large [3]. This results in a glasma with negative longitudinal pressure in the beginning of heavy ion collisions. The negative longitudinal pressure can be understood as the attraction between debris of nucleus after collisions against the deviating. Longitudinal fields are generated from the color charge on the transverse plan after collisions as shown on the right panel of figure 1 .

The evolution of the strongly coherent boost invariant color fields with negative longitudinal pressure is studied on a real time lattice simulation [8]. The initial condition is chosen according to the leading order CGC calculation, such that $T_{\mu \nu}=\operatorname{diag}(\epsilon, \epsilon, \epsilon,-\epsilon)$ for $\tau \approx 0$. The result is shown in figure 2 for a very weak coupling $g=0.1$. It is found that the coherence of fields is washed out in a very short time, and the longitudinal pressure turns into positive at $\tau \sim 1 / Q_{s}$ and approaches a constant longitudinal to transverse pressure ratio of $P_{L} / P_{T} \approx 0.1$ afterwards.

The 'bottom-up' thermalization scenario starts when the color fields are not coherent any more and quasi-particle description sets in, and occupancy of the system remains at the order of $f \sim 1 / \lambda$, i.e., for the time of $\tau \gtrsim 1 / Q_{s}$ with characteristic transverse momentum $Q_{s}$. As discussed in ref. [2], the overoccupied system will go through three stages before thermalization, as shown in figure 3 . 


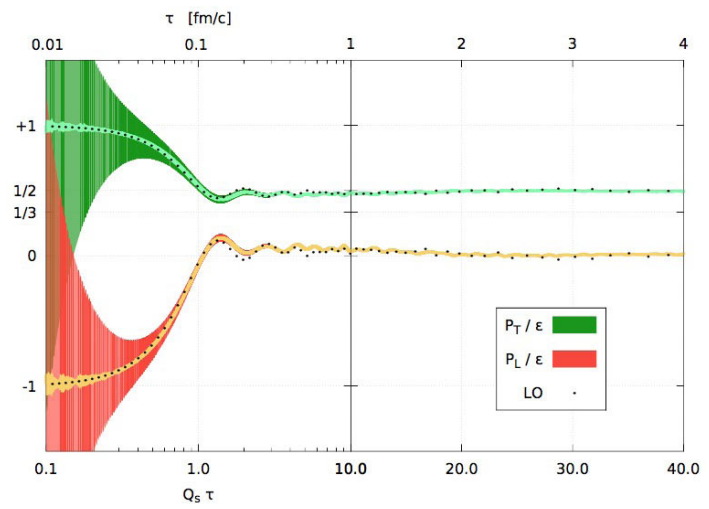

Figure 2. Time evolution of transverse $P_{T}$ and longitudinal $P_{L}$ pressure divided by energy density $\epsilon$ for $g=0.1$ right after collisions (figure taken from [8]). The initial condition is the leading order CGC calculation of $T_{\mu \nu}=$ $\operatorname{diag}(\epsilon, \epsilon, \epsilon,-\epsilon)$.

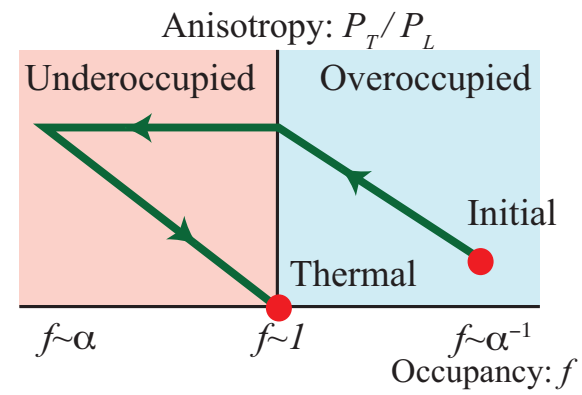

Figure 3. Three stages of bottom-up thermalization: it starts to be more anisotropic and less occupied, and reaches a maximum anisotropy at the end of the first stage. Thermalization is approaching only when radiational breakup happens in the last stage.

In the presence of longitudinal expansion, the overoccupied system moves toward more anisotropic and less occupied status in the first stage, and the maximum anisotropy is reached at $\tau \sim \lambda^{-3 / 2} / Q_{s}$. Classical fields become inappropriate to describe the system, while particle description of gluons with elastic and inelastic scattering is applicable, at the second sage for the underoccupied status. The anisotropy characterized by $P_{T} / P_{L}$ is frozen due to both the longitudinal expansion and soft particle production. The screening of the system is dominated by soft gluons, however energy is still dominated by hard partons. A thermal bath of soft gluons is formed at $\tau \sim \lambda^{-5 / 2} / Q_{s}$. In the last stage, it is the radiational breakup, with more and more hard partons (with momentum $\sim Q_{s}$ ) splitting to soft gluons and melting in the thermal bath, which drives the system toward thermalization with temperature of thermal bath increasing gradually. Full thermalization is reached at $\tau \sim \lambda^{-13 / 5}$. Hydrodynamic evolution is certainly applicable after this.

This process has been numerically simulated in CYM framework with a real time lattice configuration [9]. It is found that overoccupied systems are indeed driven toward dilute and anisotropic directions, as expected from bottom-up thermalization. Moreover, systems with different anisotropy 


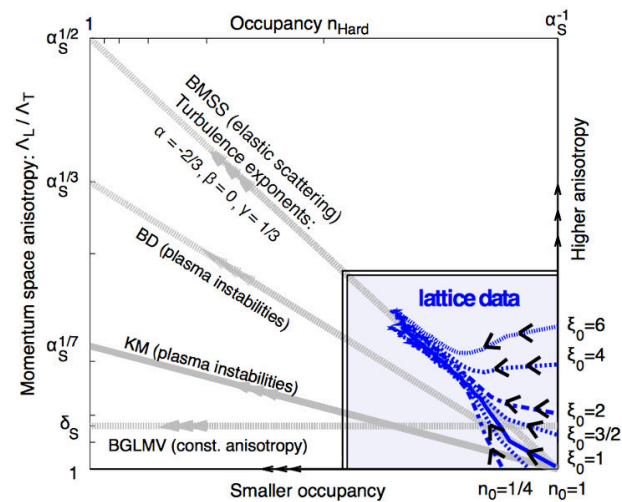

Figure 4. Universal attractor in classic Yang-Mills simulations (figure taken from [9]).

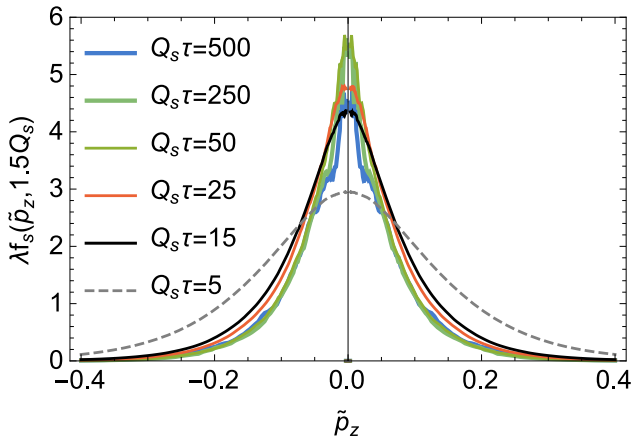

Figure 5. Scaled gluon distribution function at fixed transverse momentum $p_{\perp}=1.5 Q_{s}$, with $\tilde{p}_{z}=\left(Q_{s} \tau\right)^{1 / 3} p_{z}$ (figure taken from [10]).

and occupancy are seen to loose memory of their initial condition and flow to an universal attractor, or self-similar distribution, as shown in figure 4 , at later time. This attractor is formulated as a scaling distribution function of gluons $f\left(p_{z}, p_{\perp}, \tau\right)=\left(Q_{s} \tau\right)^{-2 / 3} f_{S}\left(\left(Q_{s} \tau\right)^{1 / 3} p_{z}, p_{\perp}\right)$, which will arise only when the exponential growth of low momentum modes from Weibel instabilities stops at $\tau \sim \ln ^{2}(1 / \lambda) / Q_{s}$ [7]. Unfortunately, as emphasised for many times, CYM theory is only valid in the first stage of bottom-up thermalization for $f \gg 1$. The effective kinetic theory turns to be the correct theory for the dynamical evolution of underoccupied system. In fact, the validity of the effective kinetic theory can be extend to overoccupied side as long as the occupancy is perturbatively small $f \ll 1 / \lambda$. The equivalence of CYM and EKT for a overoccupied system with $1 \ll f \ll 1 / \lambda$ is proven in figure 5 , where the scaling behavior form the EKT evaluation is look into through a display of the rescaled distribution function at fixed transverse momentum. The scaling behavior is reached at $\tau \sim 15 / Q_{s}$ in this example. Recalling the fact that the EKT is also applicable in the underoccupied regime, we will be able study thermalization of overoccupied systems with $1 \ll f \ll 1 / \lambda$ in weak coupling approximation.

The thermalization routes from the EKT calculation is displayed in figure 6, where the black line with $\lambda=0$ corresponds to CYM equivalent calculation, and purple dots on the line correspond to different time plotted in figure 5. Initial gluon distribution functions for different lines in figure 6 are given by

$$
\begin{aligned}
f\left(p_{z}, p_{t}\right) & =\frac{2}{\lambda} A f_{0}\left(p_{z} \xi /\left\langle p_{T}\right\rangle, p_{\perp} /\left\langle p_{T}\right\rangle\right), \\
f_{0}\left(\hat{p}_{z}, \hat{p}_{\perp}\right) & =\frac{1}{\sqrt{\hat{p}_{\perp}^{2}+\hat{p}_{z}^{2}}} e^{-2\left(\hat{p}_{\perp}^{2}+\hat{p}_{z}^{2}\right) / 3},
\end{aligned}
$$

at $Q_{s} \tau=1$, with $A$ to be chosen such that $\tau \epsilon$ is fixed. This initial condition is estimated from a 2D CYM simulation in [11] for LHC energies. In figure 6, we show two sets of longitudinal anisotropy with a few different values of ' $t$ Hooft coupling. Clearly, there is the behaviour of the bottom-up thermalization with three stages for small coupling such as $\lambda=0.5$ and 1 . The behaviour is less pronounced when the coupling increases, where thermalization (black crosses) is reached without clear distinguish of three stages. 


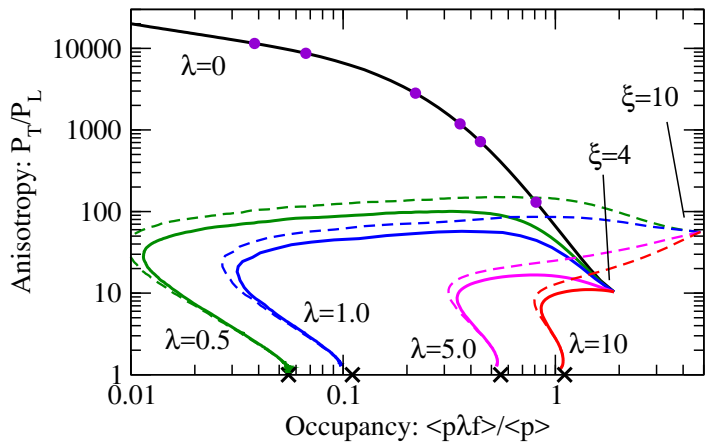

Figure 6. Thermalization routes of overoccupied system with different anisotropy parameter $\xi$ and coupling $\lambda$ in the EKT (figure taken from [10]). The black line corresponds to the calculation in classical limit. For weak coupling evolution, thermalization is marked with a black cross.

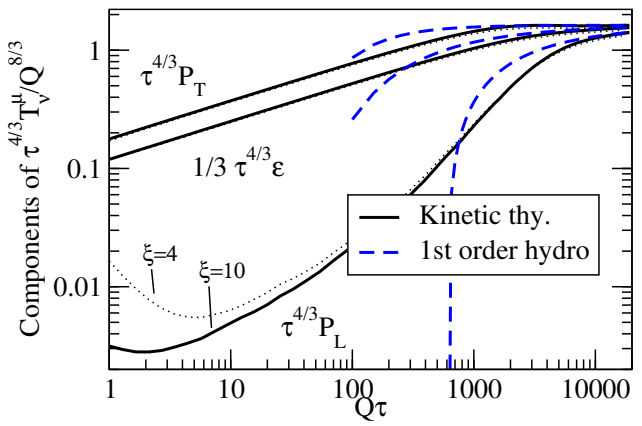

Figure 7. Time evolution of the components of energy-momentum tensor scaled with $\tau^{4 / 3} / Q^{8 / 3}$ for $\lambda=1$ (figure taken from [10]). Ideal hydro would display a flat line in this coordinate.

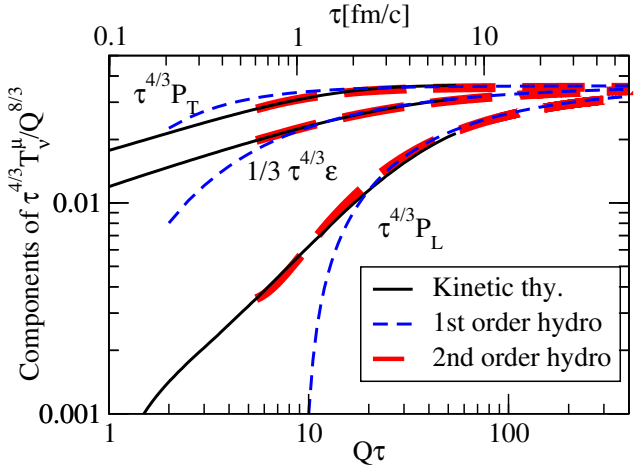

Figure 8. Time evolution of the components of energy-momentum tensor scaled with $\tau^{4 / 3} / Q^{8 / 3}$ for $\lambda=10$ (figure taken from [10]). Ideal hydro would display a flat line in this coordinate.

Viscous hydro should be approached in the last period of prethermal evolution. It is therefore interesting to compare the thermalization process with viscous hydro, where the first [12] and the second [13] order transport coefficients have been derived in perturbative QCD. Figure 7 shows time evolution of the components of energy momentum tensor scaled with $\tau^{4 / 3}$ for $\lambda=1$. In this coordinate, perfect fluid should be flat horizontal lines along the time evolution. The early time evolution of the anisotropic system behaves more like a free stream, as can be seen in figure 7. Hydrodynamics is automatically reached at very late time, as indicated by the superposition of EKT calculation and the 1 st order hydrodynamics without any free parameter. Moreover, it is known that hydrodynamization or thermalization time is parametrically proportional to $\tau \sim \lambda^{-2}$. This is confirmed in the time evolution of thermalization process for $\lambda=10$ in figure 8 , where the hydrodynamization/thermalization is approached much earlier than that for $\lambda=1$. Figure 8 has more important physical meaning as it corresponds to the relevant coupling of $\alpha_{s} \approx 0.3$ in heavy ion collision at LHC energies, where the first order hydrodynamics is reached at $\tau \sim 1 \mathrm{fm} / \mathrm{c}$ or $Q \tau \sim 10$, and it is even a bit earlier to 
reach the 2 nd order hydrodynamics. In addition, we found that viscous hydro starts to work for even large anisotropy of $P_{T} / P_{L} \approx 5$, which means that hydrodynamization happens much earlier than thermalization. This is found to be a universal feature for the thermalization at weakly and strongly coupled matter[1, 14].

\section{Conclusions}

Relativistic hydrodynamics with small viscosity has long been confirmed as the effective theory for the evolution of QGP, whereas its initial condition or the formation of QGP remains to be fully understood. Prethermal dynamics of weakly coupled glasma was discussed in this proceeding. It has been established that the prethermal evolution has a very rich history. It starts with a highly occupied and strongly coherent boost invariant fields, with weak coupling. Decoherence happens shortly after the collision $Q \tau \sim 1$, which is followed by the process of bottom-up thermalization driven by the joint influence of strongly interacting between quasi-particles and longitudinal expansion. Final state of bottom-up thermalization will provide the initial condition of hydrodynamics.

Changing of degree of freedom during the prethermal evolution results in the application of different effective theory in different processes. Solving classic Yang-Mills equation can drive the prethermal evolution to the first stage of bottom-up thermalization, which has been able to see the effect of quantum fluctuations and Weibel instabilities, and predict the universal attractor/scaling behavior at later time, but can't reach thermalization. Luckily, the effective kinetic theory bridges the gap between the first stage of bottom-up thermalization and hydrodynamics. Classical Yang-Mills theory provides a time-independent initial condition for dynamic evolution of effective kinetic theory in a regime $1 \ll f \ll 1 / \lambda$, where both of them work equally well. Based on this, bottom-up thermalization was indeed realized from the evaluation of the effective kinetic theory. Hydrodynamization is reached much earlier than thermalization. Hydrodynamization time for the relevant value of running coupling at LHC is consistent with phenomenology estimation at the order $1 \mathrm{fm} / \mathrm{c}$.

The success of hydrodynamization and thermalization in the 2+1D numerical solution of the effective kinetic theory indicates that the prethermal dynamics proceeds as our expectation. This preliminary result will inspire more interesting studies of prethermal dynamics in kinetic theory, such as the effect of anisotropic screening, fermions, and transverse dynamics, ect. Clearly, the dynamics of prethermal evolution remains an important topic for future studies.

\section{References}

[1] P. M. Chesler and L. G. Yaffe, JHEP 1510 (2015) 070 doi:10.1007/JHEP10(2015)070 [arXiv:1501.04644 [hep-th]].

[2] R. Baier, A. H. Mueller, D. Schiff and D. T. Son, Phys. Lett. B 502 (2001) 51 doi:10.1016/S03702693(01)00191-5 [hep-ph/0009237].

[3] T. Lappi and L. McLerran, Nucl. Phys. A 772 (2006) 200 doi:10.1016/j.nuclphysa.2006.04.001 [hep-ph/0602189].

[4] J. Berges, K. Boguslavski, S. Schlichting and R. Venugopalan, Phys. Rev. D 89 (2014) no.11, 114007 doi:10.1103/PhysRevD.89.114007 [arXiv:1311.3005 [hep-ph]].

[5] P. B. Arnold, G. D. Moore and L. G. Yaffe, JHEP 0301 (2003) 030 doi:10.1088/11266708/2003/01/030 [hep-ph/0209353].

[6] S. Mrowczynski, Phys. Lett. B 214 (1988) 587 Erratum: [Phys. Lett. B 656 (2007) 273]. doi:10.1016/0370-2693(88)90124-4, 10.1016/j.physletb.2007.09.039 
[7] J. Berges, B. Schenke, S. Schlichting and R. Venugopalan, Nucl. Phys. A 931 (2014) 348 doi:10.1016/j.nuclphysa.2014.08.103 [arXiv:1409.1638 [hep-ph]].

[8] T. Epelbaum and F. Gelis, Phys. Rev. Lett. $111 \quad$ (2013) 232301 doi:10.1103/PhysRevLett.111.232301 [arXiv:1307.2214 [hep-ph]].

[9] J. Berges, K. Boguslavski, S. Schlichting and R. Venugopalan, Phys. Rev. D 89 (2014) no.7, 074011 doi:10.1103/PhysRevD.89.074011 [arXiv:1303.5650 [hep-ph]].

[10] A. Kurkela and Y. Zhu, Phys. Rev. Lett. 115 (2015) no.18, 182301 doi:10.1103/PhysRevLett.115.182301 [arXiv:1506.06647 [hep-ph]].

[11] T. Lappi, Phys. Lett. B 703 (2011) 325 doi:10.1016/j.physletb.2011.08.011 [arXiv:1105.5511 [hep-ph]].

[12] P. B. Arnold, G. D. Moore and L. G. Yaffe, JHEP 0305 (2003) 051 doi:10.1088/11266708/2003/05/051 [hep-ph/0302165].

[13] M. A. York and G. D. Moore, Phys. Rev. D 79 (2009) 054011 doi:10.1103/PhysRevD.79.054011 [arXiv:0811.0729 [hep-ph]].

[14] L. Keegan, A. Kurkela, P. Romatschke, W. van der Schee and Y. Zhu, JHEP 1604 (2016) 031 doi:10.1007/JHEP04(2016)031 [arXiv:1512.05347 [hep-th]]. 